\title{
HBZ and its roles in HTLV-1 oncogenesis
}

\author{
Tiejun Zhao ${ }^{1,2}$ and Masao Matsuoka ${ }^{1}$ * \\ ' Laboratory of Virus Control, Institute for Virus Research, Kyoto University, Kyoto, Japan \\ ${ }^{2}$ College of Chemistry and Life Sciences, Zhejiang Normal University, Jinhua, China
}

\section{Edited by:}

Renaud Mahieux, École Normale

Superieure de Lyon, France

\section{Reviewed by:}

Keiji Ueda, Osaka University

Graduate School of Medicine, Japan

Rika Ann Furuta, Japanese Red Cross

Osaka Blood Center, Japan

\section{${ }^{*}$ Correspondence:}

Masao Matsuoka, Laboratory of Virus Control, Institute for Virus

Research, Kyoto University, 53

Shogoin Kawahara-cho, Sakyo-ku,

Kyoto 606-8507, Japan.

e-mail:mmatsuok@virus.kyoto-u.ac.jp
Human T-cell leukemia virus type 1 (HTLV-1) causes adult T-cell leukemia (ATL). The minus strand of HTLV-1 provirus encodes a bZIP protein donated as HTLV-1 bZIP factor (HBZ). Among the HTLV-1 regulatory and accessory genes, the tax and HBZ genes were thought to play critical roles in oncogenesis. However, $\mathrm{HBZ}$ is the only gene that remains intact and is consistently expressed in all ATL cases, while the tax gene is frequently inactivated by epigenetic modifications or deletion of the $5^{\prime} L T R$. HBZ gene promotes the proliferation of ATL cells through its mRNA form. Moreover, HBZ induces T-cell lymphoma and systemic inflammation in vivo. HBZ fulfills its functions mainly through regulating HTLV-1 5'LTR transcription and modulating a variety of cellular signaling pathways which are related with cell growth, immune response, and T-cell differentiation. Taken together, the multiple functions of HBZ render its predominant function in leukemogenesis of ATL.

Keywords: HBZ, HTLV-1, Tax, viral oncogenesis, regulatory T cells

\section{INTRODUCTION}

Human T-cell leukemia virus type 1 (HTLV-1) was the first retrovirus proven to be associated with human disease. The infection of HTLV-1 causes adult T-cell leukemia (ATL) and chronic inflammatory diseases including HTLV-1-associated myelopathy (HAM)/tropical spastic paraparesis (TSP; Uchiyama et al., 1977; Poiesz et al., 1980). HTLV-1 belongs to deltaretrovirus family, which include HTLV-2, HTLV-3, and HTLV-4; the simian Tcell lymphotropic viruses (STLV-1, STLV-2, and STLV-3) and the bovine leukemia virus (BLV; Matsuoka and Jeang, 2007). Like other retroviruses, the HTLV-1 proviral genome has structural genes, gag, pol, and env, flanked by long terminal repeat (LTR) at both ends (Figure 1; Matsuoka and Jeang, 2007). The characteristic of HTLV-1 proviral genome is the presence of $\mathrm{pX}$ region between env and $3^{\prime} \mathrm{LTR}$, and encoded several accessory genes, which include tax, rex, p12, p21, p30, p13, and HTLV-1 bZIP factor (HBZ; Gaudray et al., 2002; Nicot et al., 2005). HBZ was first identified as an antisense viral protein that associated with cAMP-response element binding protein-2 (CREB-2) in HTLV-1infected cells (Gaudray et al., 2002). We found that the expression of HBZ is conserved in all ATL cells (Satou et al., 2006), and nonsense mutations of all HTLV-1 genes except the HBZ gene were generated byAPOBEC3G before integration of the provirus (Fan et al., 2010). In addition, the $H B Z$ gene promotes the proliferation of ATL cells (Satou et al., 2006). Thus, the HBZ gene is believed to be essential for leukemogenesis. In this review, we discuss the updated understanding of HTLV- 1 and focus on the roles of HBZ in HTLV-1 oncogenesis.

\section{STRUCTURE OF HBZ}

The existence of a viral transcript encoded by the minus strand of the HTLV-1 provirus was reported in 1989 (Larocca et al., 1989). Thereafter, a viral protein that binds to CREB-2 was identified by yeast 2-hybrid screening system, and termed as HTLV-1 bZIP factor (HBZ; Gaudray et al., 2002). The 5'RACE experiment identified the transcription start sites of HBZ were located in the U3 and R regions of the HTLV-1 3'LTR (Satou et al., 2006). 5'LTR of HTLV-1 was frequently lost and methylated in ATL cases, while 3'LTR was not deleted and remained unmethylated (Matsuoka and Jeang, 2007). It may be one of the reasons why HBZ is consistently expressed in all ATL cells. Two transcripts of the $H B Z$ gene have been revealed: a spliced form ( $\mathrm{sHBZ}$ ) and an unspliced form (usHBZ; Figure 1). The promoter of the spliced and unspliced HBZ transcripts was TATA-less and contained initiators and downstream promoter elements (Yoshida et al., 2008). Detailed analyses of the promoter of $\mathrm{sHBZ}$ gene showed that $\mathrm{Sp} 1$ sites were critical for its transcription. U3 region of LTR in the both end of HTLV1 proviral genome contains Tax-response element (TRE), which acts as an enhancer of viral transcription. Tax and CREB can activate transcription of the HBZ gene through $3^{\prime} \mathrm{LTR}$, meanwhile, Tax regulated antisense promoter activity is influenced by the integration site. However, Tax induced $3^{\prime}$ LTR activation is not as significant as it did on the transcription of the HTLV-1 plus strand (Landry et al., 2009).

The spliced and unspliced $H B Z$ genes are translated into a polypeptide of 206 and 209 amino acids, respectively. Both forms of HBZ contain three domains: N-terminal activation domain $(\mathrm{AD})$, central domain $(\mathrm{CD})$, and basic ZIP domain (bZIP) in the C-terminal (Figure 2). There are two LXXLL-like motifs located within the N-terminal AD domain of HBZ. Both are important for the binding to p300/CBP (Clerc et al., 2008). The HBZ protein is localized in the nucleus with a speckled pattern. Three nuclear localization signals (NLSs) were identified to two regions in the CD of HBZ and a basic region of the bZIP domain (Hivin et al., 2005). Moreover, the integrity of HBZ protein sequence was necessary for its accumulation in nuclear speckles. HBZ specifically distributed in the nucleus to heterochromatin, while it is not associated with heterochromatin (Hivin et al., 2005). 


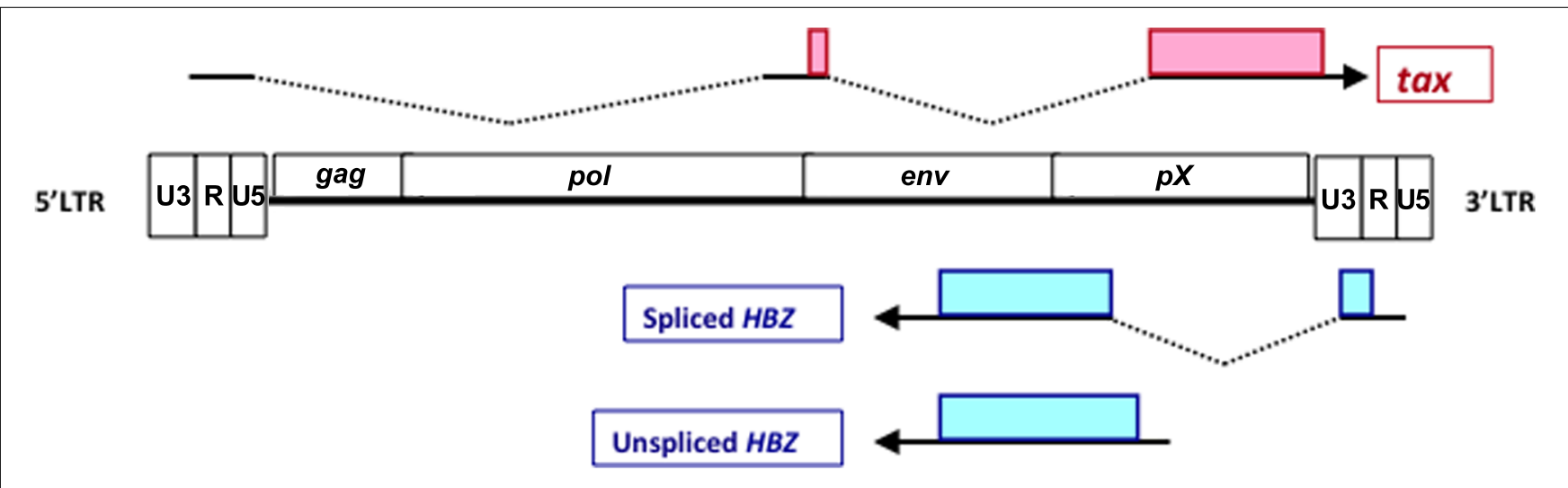

FIGURE 1 | Schematic diagram of spliced HBZ, unspliced HBZ and Tax. Tax is encoded by the positive strand of HTLV-1 proviral genome. HBZ is located on the complementary strand and transcribed from 3'LTR. Spliced and unspliced HBZ are showed. Shaded boxes represent their coding regions.

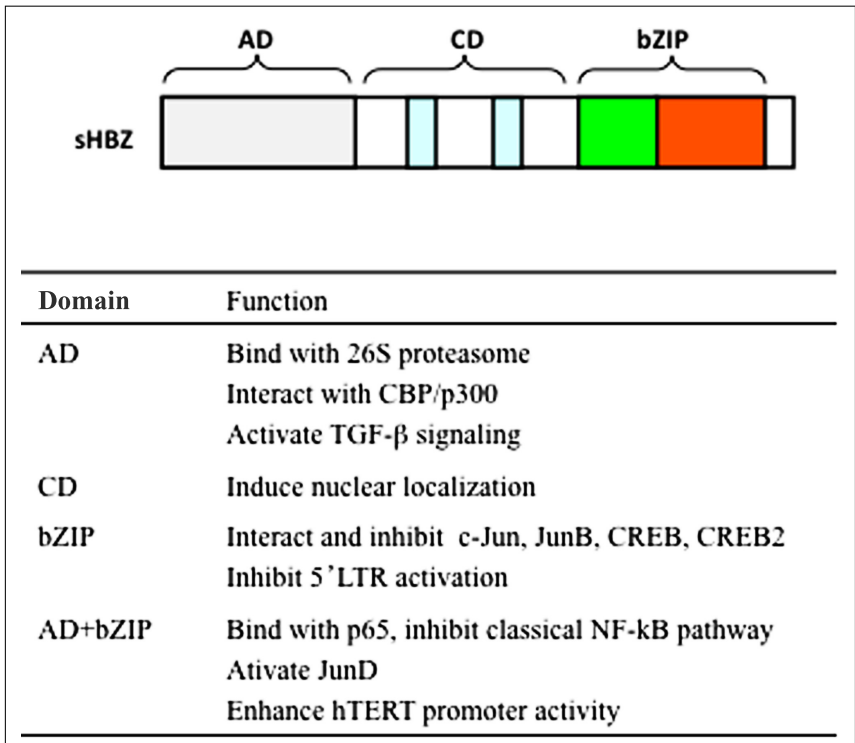

FIGURE 2 | Schematic diagram of HBZ domains. HBZ has three domains: activation domain (AD), central domain (CD), and basic ZIP domain (bZIP). Functions of each domain are listed.

The only difference between the protein form of sHBZ and usHBZ are seven amino acids in their N-terminal, while it causes significant difference between these two proteins. The half-life of usHBZ protein is much shorter than that of $\mathrm{sHBZ}$ (Yoshida et al., 2008). In ATL cell, only sHBZ protein could be detected, since $\mathrm{SHBZ}$ gene is more predominant than us $H B Z$ gene (Usui et al., 2008).

\section{TRANSCRIPTION OF THE HBZ GENE}

The transcription of HTLV-1 viral gene is the symbol of HTLV1 infection. Among all of the HTLV transcripts, the tax gene is thought to play critical role in leukemogenesis because of its pleiotropic functions. However, about $60 \%$ of ATL cases lost the tax expression by three mechanisms: (1) deletion of HTLV-1 5'LTR, (2) hypemethylation of 5'LTR, and (3) non-sense mutation, deletion, and insertion of tax gene (Matsuoka and Jeang, 2007). Moreover, the loss of Tax is believed to occur during the process of oncogenesis. It indicates that Tax expression is not always necessary for ATL. Unlike 5'LTR, HTLV-1 3'LTR is intact and remains unmethylated. The $H B Z$ gene expression was conserved in all ATL cases. In addition, expression of the $H B Z$ gene could be detected not only in ATL cells but also in HTLV-1-infected carriers, furthermore, its expression are well correlated with proviral load. It has been reported the correlation between the level of the $H B Z$ gene and severity of HAM/TSP, indicating that HBZ might contribute to the development of HAM/TSP except for ATL (Saito et al., 2009).

The two forms of the $H B Z$ gene are not equally expressed in ATL cells. The expression level of the $s H B Z$ gene was found to be four times higher than that of the us $H B Z$ gene transcript in HTLV- 1 carriers and ATL cases.

In PBMCs from HTLV-1-infected rabbits revealed that the tax gene was expressed at the highest levels immediately after infection and progressively decreased, eventually stabilizing at low levels. However, $H B Z$ was detectable at low levels early after infection, and slowly increased and stabilized. At 8 weeks post infection, the $H B Z$ mRNA was expressed at the highest concentration of all mRNA measured (an average of ninefold more than tax mRNA; Li et al., 2009). Additionally, Suemori et al. (2009) reported that the expression levels of HBZ mRNA and HBZ protein in HTLV-1-infected cells were not parallel, suggest that the post transcriptional regulation of $H B Z$ gene may differ among different cell types. However, other group detected the consistently high level of HBZ mRNA and protein in ATL- and HTLV-1-associated cell lines (Satou et al., 2006; Zhao etal., 2011). The physiological level of HBZ could regulate the cellular signaling pathway, which is critical for T-cell differentiation. The discrepancy between two independent studies may due to the different experiment condition and different HBZ antibody they used.

\section{THE BIOLOGICAL FUNCTION OF HBZ INHIBITION OF TAX-MEDIATED VIRAL TRANSCRIPTION}

HBZ was firstly identified as a CREB-2 binding protein which could inhibit HTLV-1 transcription from 5'LTR. Further study 
showed the association between HBZ and CREB (Lemasson et al., 2007). HBZ bZIP domain is responsible for its interacting with CREB-2/CREB. These interactions abolished the binding of CREB-2/CREB to the Tax-responsive element (TxRE) in the HTLV-1 5'LTR and the cyclic AMP-response element (CRE) in cellular promoters, resulting in the suppression of Tax-mediated transcription from 5'LTR (Gaudray et al., 2002; Lemasson et al., 2007). In addition, HBZ interacts with CBP/p300 co-activator and suppressed the transcription from the $5^{\prime}$ LTR by Tax (Clerc et al., 2008). Through two LXXLL-like motifs in the N-terminal AD, $\mathrm{HBZ}$ binds with CBP/p300 KIX domain, inhibiting the recruitment of CBP/p300 to the HTLV-1 promoter. Further study found that HBZ-AD interacts with the MLL binding surface of KIX domain (Cook et al., 2011).

\section{PROLIFERATION OF ATL CELLS}

Stable expression of HBZ maintains the growth of Kit 225 cells (Satou et al., 2006). When knock down the expression of HBZ in ATL cell lines, MT-1 and TL-Om1, by siRNA, their proliferation was suppressed (Satou et al., 2006). It indicated that HBZ is essential for the continuous expansion of ATL cells. Furthermore, studies of the growth of T-cell lines transfected with the mutated $H B Z$ genes showed that HBZ promotes T-cell proliferation in its mRNA form, whereas HBZ suppresses Tax-mediated viral transcription via protein form. Mutation analyses of the $H B Z$ gene showed that $H B Z$ RNA promotes cell proliferation by forming secondary stem-loop structures. $H B Z$ RNA activates transcription of E2F1 and its target genes, and increases G1/S transition (Satou et al., 2006).

Spliced HBZ could induce the proliferation of ATL cells, while usHBZ could not (Yoshida et al., 2008). It suggests that the first exon of the sHBZ transcript is critical for its growth promoting activity. The first exon of HBZ corresponds to the Rex-responsive element (RxRE) of the HTLV-1 3'LTR. It is known that Rex promotes the export of viral RNA with an RxRE region. Together, we speculate that $s H B Z$ RNA form stem-loop structure, which different as usHBZ RNA, inducing the proliferation of ATL cells.

ATF3, an HBZ binding protein, was highly expressed in ATL cells. ATF3 expression has positive and negative effects on the proliferation and survival of ATL cells. HBZ impairs its negative effects, leaving ATF3 to promote proliferation of ATL cells via mechanisms including upregulation of CDC2 and cyclin E2. HBZ may take it as advantages to promote the cell survive (Hagiya et al., 2011).

\section{MODIFICATION OF AP-1 SIGNALING}

Previous studies show that the interaction between bZIP proteins affects their cellular localization and modulates transcriptional activity. Activator protein-1 (AP-1) functions as a dimer comprised of bZIP proteins of Jun family (c-Jun, JunB, JunD), Fos family (c-Fos, FosB, Fra-1, Fra-2), and others. HBZ interacted with c-Jun/JunB and repressed c-Jun/JunB mediated signaling by abrogating its DNA-binding capacity. HBZ induced the degradation of c-Jun protein through a proteasome-dependent pathway, while it is in a ubiquitin-independent manner (Basbous et al., 2003; Matsumoto et al., 2005; Isono et al., 2008). In addition, HBZ inhibited AP-1 signaling by sequestrating c-Jun in nuclear bodies.
On the contrary, HBZ can activate transcription mediated by JunD via forming heterodimers with JunD (Thebault et al., 2004). HBZ/JunD heterodimers interact with Sp1 transcription factors and enhanced the transcription of telomerase reverse transcriptase (hTERT; Thebault et al., 2004). Since telomerase activation is thought to be associated with tumor malignant, the induction of hTERT by HBZ may be implicated in the oncogenesis of ATL.

\section{SUPPRESSION OF THE CLASSIC NF- $\kappa B$ PATHWAY}

In ATL, Tax-mediated nuclear factor- $\kappa \mathrm{B}(\mathrm{NF}-\kappa \mathrm{B})$ activation plays an important role in the proliferation of ATL cells and in the transforming activity of HTLV-1 (Yamaoka et al., 1996). Activation of NF- $\kappa \mathrm{B}$ by Tax includes both the classical and alternative pathways. Contrary, HBZ selectively suppresses the p65 mediated classical NF- $\kappa \mathrm{B}$ pathway without inhibiting alternative NF- $\kappa \mathrm{B}$ signaling (Zhao et al., 2009). There are two mechanisms involved in this inhibition. One mechanism is that HBZ inhibits p65 DNA-binding ability via interacting with p65. Another is that HBZ increases the expression of PDLIM2, an E3 ubiquitin ligase for p65, leading to the increase of p65 ubiquitination and degradation (Zhao et al., 2009). Both activation and bZIP domains are necessary for the inhibition of classic NF- $\mathrm{B}$ pathway by HBZ. The selective modulation of classical NF- $\kappa \mathrm{B}$ pathway by $\mathrm{HBZ}$ might explain the predominant activation of alternative pathway, and perhaps to oncogenesis. PDLIM2 has been reported to suppress Tax-mediated tumorigenesis by targeting Tax into nuclear matrix for proteasomal degradation (Yan et al., 2009). Together, this may offer an explanation for the repression of Tax in ATL cells, by which HBZ activates the expression of PDLIM2 and PDLIM2 subsequently functions to degrade Tax. The updated report showed that HBZ inhibits the acetylation of p65. It may contribute to the repression of classical NF- $\kappa$ B pathway by HBZ.

Recent studies suggest that the classical NF-кB pathway is mostly involved in innate immunity and inflammatory responses. The inhibition of NF-кB pathway is common among different viruses, suggesting that these activities are important for viral survival and infection. HTLV-1 might facilitate escape from the host immune attack by suppressing the classical NF- $\kappa$ B pathway by HBZ.

\section{INDUCED TREG DIFFERENTIATION THROUGH ENHANCING TGF- $\beta$ SIGNALING}

Adult T-cell leukemia cells, present a $\mathrm{CD} 4{ }^{+} \mathrm{CD} 25^{+}$phenotype, have the same markers as those of natural regulatory $\mathrm{T}$ cells (Tregs; Matsuoka and Jeang, 2007). Expression of FoxP3 was detected in two-thirds of ATL cases, indicating that ATL may originates from natural Treg cells infected with HTLV-1 (Karube et al., 2004). Recently, we found that transgenic expression of HBZ increases Foxp $3^{+}$Treg cells and effector/memory $\mathrm{T}$ cells, leading to development of T-cell lymphomas and systemic inflammatory diseases (Satou et al., 2011). It is well known that TGF- $\beta$ signaling is critical for the development of Treg. Our study showed that HBZ enhanced TGF- $\beta$ signaling in a p300-dependent manner (Zhao et al., 2011). HBZ, Smad3, and p300 formed a ternary complex, and the association between Smad3 and p300 was enhanced in the presence of HBZ. HBZ 
could overcome the suppressive effect of Tax on TGF- $\beta$ pathway. The enhancement of TGF- $\beta$ signaling by HBZ leads to the up-regulation of Foxp3 in naïve T cells (Zhao et al., 2011). This might account for why HTLV-1 infection increases Tregs in vivo. Interestingly, $\mathrm{HBZ}$ maintains the cell growth even with the presence of TGF- $\beta$, and HBZ does not influence the expression of genes associated with cell cycle and proliferation. It indicates HBZ selectively modulates actions of TGF- $\beta /$ Smad signaling pathway.

By enhancing TGF- $\beta$ pathway and Foxp3 expression, HBZ enables HTLV-1 to convert infected T cells into Tregs, which is thought to be a critical strategy for virus persistence.

\section{PATHOGENIC FUNCTION OF HBZ}

Tax is recognized as an oncoprotein, since it immortalizes primary $\mathrm{T}$ cells and confers proliferative properties to HTLV-1-infected cells. However, transcript of the tax gene is detected in only $40 \%$ of ATL cases. In Tax transgenic mice, cancers were induced depending on the promoter used (Lairmore et al., 2005). To better understand the pathogenic function of HBZ, we generated transgenic mice expressing HBZ under mouse CD4 promoter and enhancer. The HBZ transgenic mice (HBZ-Tg) present many similarities in symptoms and immunological features with HTLV-1-infected individuals (Satou et al., 2011). In the total splenocytes of HBZ transgenic mice, the percentage of CD4-positive T cells increased. In addition, HBZ promotes the proliferation of thymocytes from transgenic mice. More than one third of HBZ-Tg mice developed T-cell lymphomas after a long latent period. To study the underlying molecular mechanisms, we analyzed the phenotype and function of $\mathrm{T}$ cells and found $\mathrm{CD} 4^{+} \mathrm{Foxp}^{+}$Tregs and effector/memory $\mathrm{CD} 4^{+} \mathrm{T}$ cells increased in vivo. The high percentage of Foxp $3^{+}$cells in HBZ-Tg mice is also observed in lymphoma tissues from human ATL patients. However, the increased CD4 ${ }^{+} \mathrm{Foxp}^{+}$ Treg cells in HBZ transgenic mice were functionally impaired since HBZ could physically interact with Foxp3 and NFAT, thereby impairing the suppressive function of Treg cells (Satou et al., 2011). In addition, most of HBZ transgenic mice spontaneously develop inflammatory lesions in the skin and lung, and $\mathrm{CD}^{+}{ }^{+} \mathrm{CD} 4^{+} \mathrm{T}$ cells infiltrated into the dermis and epidermis in the lesions. These findings are similar to those observed in HTLV-1 carriers.

\section{IMPAIRED CELL-MEDIATED IMMUNITY}

Viruses have strategies to escape the host immune attack and to replicate in vivo. HTLV-1 infection is complicated by opportunistic infections. However, it was still unknown how HTLV-1 causes immune deficiency. Recently, we found that HBZ transgenic mice were more vulnerable to the infections of herpes simplex virus type 2 (HSV-2) and Listeria monocytogenes (LM) than non-Tg mice (Sugata et al., 2012). The production of Th1 cytokines, such as IFN- $\gamma$, IL-2, and TNF- $\alpha$, was suppressed in HBZ-Tg mice. Moreover, the impaired production of IFN- $\gamma$ is also observed in primary CD4 T cells from ATL patients. This study has shown that HBZ suppresses the IFN- $\gamma$ promoter activity through suppressing NFAT and AP-1 signaling pathways (Sugata et al., 2012). Together, it is the first report shown that HBZ inhibits CD4 T-cell responses, resulting in impaired host immunity in vivo.

\section{THE RELATIONSHIP BETWEEN TAX AND HBZ}

Accumulating evidence showed that Tax and HBZ are two important factors for ATL. The crossover and the interaction between these two proteins are important for the HTLV-1 oncogenesis. Tax could transform rodent cells and immortalizes human primary $\mathrm{T}$ cells, moreover, it enhances viral replication, supports cellular proliferation, inhibits apoptosis, impairs cell-cycle checkpoints, and induces DNA damages. Although Tax has pleiotropic functions as mentioned above, $\sim 60 \%$ of all ATL cases did not express Tax. Moreover, abortive genetic changes, including deletions, insertions, and non-sense mutations, were frequent in all viral genes include the tax gene. Non-sense mutations in the tax gene were also observed in proviruses from asymptomatic carriers. Contrary to the tax gene, HBZ is consistently expressed in all ATL cells and remains intact through the whole period of ATL development. Tax plays a central role in the immortalization of T-lymphocytes in cell culture, on the contrary, HBZ was dispensable for viral-induced immortalization of primary human T cells (Arnold et al., 2006). Tax is the major target of cytotoxic T-lymphocytes (CTLs) in vivo (Kannagi et al., 1991), while HBZ is not a target antigen for HTLV-1-specific CTLs (Suemori et al., 2009). Since HBZ promote the T-cell proliferation and induced lymphoma and chronic inflammation which is similar to those in HTLV-1-infected individuals, we believe HBZ play more important role in the maintenance of HTLV-1-induced transformation.

Interestingly, Tax and HBZ have opposite effect on the regulation of cellular signaling pathways (Table 1). Tax activates 5 LTR transcription; enhances AP-1 and classic NF- $\kappa \mathrm{B}$ signaling, and suppresses TGF- $\beta$ pathway. Contrary, HBZ suppressed Tax-mediated HTLV-1 viral transcription; inhibit activated AP1 and classical NF- $\kappa$ B signaling. However, HBZ enhances TGF- $\beta$ pathway. HTLV-1 may take advantage of this opposite function of Tax and $\mathrm{HBZ}$ on regulating signaling pathway to allowing the better survive of HTLV-1-infected cells and escape the immune surveillance.

Table 1 | Character and regulatory function of Tax and HBZ.

\begin{tabular}{|c|c|c|}
\hline & $\operatorname{Tax}$ & HBZ \\
\hline Expression in ATL cells & $\sim 40 \%$ & $100 \%$ \\
\hline Contain genetic changes & Yes & No \\
\hline Function as mRNA & ND & Yes \\
\hline Immortalize T cells & Yes & No \\
\hline Target by CTL & Yes & No \\
\hline HTLV-1 5'LTR transcription & Activate & Inhibit \\
\hline Classical NF-кB pathway & Activate & Inhibit \\
\hline Alternative NF-кB pathway & Activate & No \\
\hline AP-1 activity & Activate & Inhibit \\
\hline JunD transcription & Activate & Activate \\
\hline T-cell proliferation & Activate & Activate \\
\hline TGF- $\beta$ signaling & Inhibit & Activate \\
\hline Host immune response & Enhance & Suppress \\
\hline
\end{tabular}




\section{ANTISENSE TRANSCRIPT OF RETROVIRUS}

There are four kinds of HTLVs have been identified, and their oncogenic property is different. HTLV-1 mainly infected CD4 ${ }^{+}$ $\mathrm{T}$ cells. However, HTLV-2 tends to target $\mathrm{CD} 8^{+} \mathrm{T}$ cells, and induces cancer only in rare cases. Unlike Tax1, Tax2 which encodes by HTLV-2 does not have PDZ domain-binding motif (PBM). Since PBM is important for the transforming activity, such differences between Tax 1 and Tax 2 may responsible for the different pathogenicities of HTLV-1 and HTLV-2 (Hirata et al., 2004). It was reported that HTLV-2 produced an antisense transcript termed antisense protein (ASP) of HTLV-2 (APH-2; Figure 3; Halin et al., 2009). Like the $s H B Z$ gene, $A P H-2$ mRNA is transcribed from the $3^{\prime} \mathrm{LTR}$, and spliced and polyadenylated. APH-2 does not contain bZIP domain but it still could interact with CREB and suppresses Tax2-dependent LTR activation. In addition, APH-2 did not colocalize with the nucleolus, although in non- $\mathrm{T}$ and $\mathrm{T}$ cells, it did have nuclear localization. Importantly, APH-2 was correlated with proviral load, while it does not promote cell proliferation and does not cause lymphocytosis.

Recent reports showed that HTLV-3 and HTLV-4 produce a spliced and polyadenylated antisense transcript (Barbeau and Mesnard, 2011). These two proteins were named as APH-3 and APH-4, respectively, since both proteins lack consensus bZIP domain (Figure 3). But, they retained the capacity to inhibit LTR activation that mediated by their respective Tax proteins. This study demonstrated a nuclear-restricted pattern for APH4 while APH-3 was localized both in the cytoplasm and in the nucleus.

A naturally occurring antisense RNA was identified in the cell lines infected with HIV-1, and encodes an ASP with an apparent molecular mass of $19 \mathrm{kDa}$ (Figure 3; Vanhee-Brossollet et al., 1995; Clerc et al., 2011). Antibodies against this protein have been detected in the sera of HIV-positive individuals. However, it remains to be elucidated the function of this ASP in vivo.

\section{PERSPECTIVES}

Intensive studies on the leukemogenesis of ATL since the discovery of HTLV-1 have revealed some molecular mechanisms by which viral proteins induce viral replication and cellular transformation. However, the precise mechanisms of oncogenesis by HTLV-1 remain to be clarified. Recent progresses on the researches of the $H B Z$ gene give new ideas into the mechanisms of oncogenesis. HBZ is the only viral gene that is expressed in all ATL

\section{REFERENCES}

Arnold, J., Yamamoto, B., Li, M., Phipps, A. J., Younis, I., Lairmore, M. D., and Green, P. L. (2006). Enhancement of infectivity and persistence in vivo by HBZ, a natural antisense coded protein of HTLV-1. Blood 107, 3976-3982.

Barbeau, B., and Mesnard, J. M. (2011). Making sense out of antisense transcription in human T-cell lymphotropic viruses (HTLVs). Viruses 3 , 456-468.

Basbous, J., Arpin, C., Gaudray, G., Piechaczyk, M., Devaux, C., and
Mesnard, J. M. (2003). The HBZ factor of human T-cell leukemia virus type I dimerizes with transcription factors JunB and c-Jun and modulates their transcriptional activity. J. Biol. Chem. 278, 43620-43627.

Clerc, I., Laverdure, S., Torresilla, C., Landry, S., Borel, S., Vargas, A., Arpin-Andre, C., Gay, B., Briant, L., Gross, A., Barbeau, B., and Mesnard, J. M. (2011). Polarized expression of the membrane ASP protein derived from HIV-1 antisense transcription in T cells. Retrovirology 8, 74 .

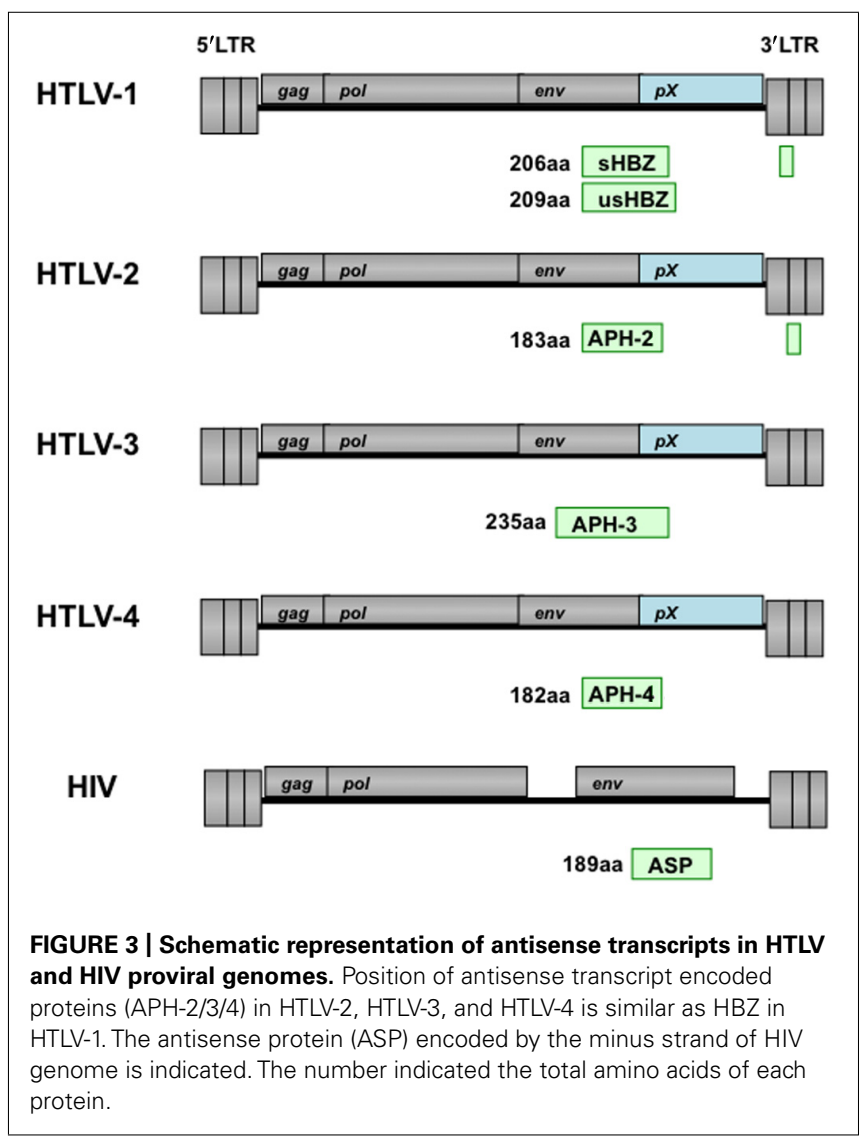

cases. Except the $H B Z$ gene, non-sense mutations of other viral genes are detected before integration of provirus. Importantly, HBZ maintains the cell survival when Tax is silenced. These data collectively indicate that HBZ protein has predominate function on the oncogenesis of HTLV-1. Further studies are needed to clarify the oncogenic function of HBZ and develop therapy targeted against HBZ based on the growing knowledge of HTLV-1 molecular biology.

\section{ACKNOWLEDGMENTS}

This work was supported by a Grant-in-aid for Scientific Research from the Ministry of Education, Science, Sports, and Culture of Japan, and the Sciences Foundation of Zhejiang Normal University.

Clerc, I., Polakowski, N., Andre-Arpin, C., Cook, P., Barbeau, B., Mesnard, J. M., and Lemasson, I. (2008). An interaction between the human $\mathrm{T}$ cell leukemia virus type 1 basic leucine zipper factor (HBZ) and the KIX domain of p300/CBP contributes to the down-regulation of tax-dependent viral transcription by HBZ. J. Biol. Chem. 283, 2390323913.

Cook, P. R., Polakowski, N., and Lemasson, I. (2011). HTLV-1 HBZ protein deregulates interactions between cellular factors and the KIX domain of p300/CBP. J. Mol. Biol. 409, 384-398.

Fan, J., Ma, G., Nosaka, K., Tanabe, J., Satou, Y., Koito, A., WainHobson, S., Vartanian, J. P., and Matsuoka, M. (2010). APOBEC3G generates nonsense mutations in human T-cell leukemia virus type 1 proviral genomes in vivo. J. Virol. 84, 7278-7287.

Gaudray, G., Gachon, F., Basbous, J., Biard-Piechaczyk, M., Devaux, C., and Mesnard, J. M. (2002). The complementary strand of the human T-cell leukemia virus type 1 RNA 
genome encodes a bZIP transcription factor that down-regulates viral transcription. J. Virol. 76, 12813-12822.

Hagiya, K., Yasunaga, J., Satou, Y., Ohshima, K., and Matsuoka, M. (2011). ATF3, an HTLV-1 bZip factor binding protein, promotes proliferation of adult T-cell leukemia cells. Retrovirology 8, 19.

Halin, M., Douceron, E., Clerc, I., Journo, C., Ko, N. L., Landry, S., Murphy, E. L., Gessain, A., Lemasson, I., Mesnard, J. M., Barbeau, B., and Mahieux, R. (2009). Human T-cell leukemia virus type 2 produces a spliced antisense transcript encoding a protein that lacks a classic bZIP domain but still inhibits Tax2mediated transcription. Blood 114, 2427-2438.

Hirata, A., Higuchi, M., Niinuma, A., Ohashi, M., Fukushi, M., Oie, M., Akiyama, T., Tanaka, Y., Gejyo, F., and Fujii, M. (2004). PDZ domain-binding motif of human Tcell leukemia virus type 1 Tax oncoprotein augments the transforming activity in a rat fibroblast cell line. Virology 318, 327-336.

Hivin, P., Frederic, M., Arpin-Andre, C., Basbous, J., Gay, B., Thebault, S., and Mesnard, J. M. (2005). Nuclear localization of HTLV-I bZIP factor (HBZ) is mediated by three distinct motifs. J. Cell Sci. 118, 1355-1362.

Isono, O., Ohshima, T., Saeki, Y., Matsumoto, J., Hijikata, M., Tanaka, K., and Shimotohno, K. (2008). Human T-cell leukemia virus type $1 \mathrm{HBZ}$ protein bypasses the targeting function of ubiquitination. J. Biol. Chem. 283, 34273-34282.

Kannagi, M., Harada, S., Maruyama, I., Inoko, H., Igarashi, H., Kuwashima, G., Sato, S., Morita, M., Kidokoro, M., Sugimoto, M., Funahashi, S.-I., Osame, M., and Shida, H. (1991). Predominant recognition of human T cell leukemia virus type I (HTLV-I) pX gene products by human $\mathrm{CD}^{+}$ cytotoxic $\mathrm{T}$ cells directed against HTLV-I-infected cells. Int. Immunol. 3, 761-767.

Karube, K., Ohshima, K., Tsuchiya, T., Yamaguchi, T., Kawano, R., Suzumiya, J., Utsunomiya, A., Harada, M., and Kikuchi, M. (2004). Expression of FoxP3, a key molecule in CD4CD25 regulatory $\mathrm{T}$ cells, in adult T-cell leukaemia/lymphoma cells. Br. J. Haematol. 126 , 81-84.
Lairmore, M. D., Silverman, L., and Ratner, L. (2005). Animal models for human T-lymphotropic virus type 1 (HTLV-1) infection and transformation. Oncogene 24, 6005-6015.

Landry, S., Halin, M., Vargas, A., Lemasson, I., Mesnard, J. M., and Barbeau, B. (2009). Upregulation of human Tcell leukemia virus type 1 antisense transcription by the viral tax protein. J. Virol. 83, 2048-2054.

Larocca, D., Chao, L. A., Seto, M. H., and Brunck, T. K. (1989). Human T-cell leukemia virus minus strand transcription in infected T-cells. Biochem. Biophys. Res. Commun. 163, 10061013.

Lemasson, I., Lewis, M. R., Polakowski, N., Hivin, P., Cavanagh, M. H., Thebault, S., Barbeau, B., Nyborg, J. K., and Mesnard, J. M. (2007). Human T-cell leukemia virus type 1 (HTLV1) bZIP protein interacts with the cellular transcription factor CREB to inhibit HTLV-1 transcription. J. Virol. 81, 1543-1553.

Li, M., Kesic, M., Yin, H., Yu, L., and Green, P. L. (2009). Kinetic analysis of human T-cell leukemia virus type 1 gene expression in cell culture and infected animals. J. Virol. 83, 3788-3797.

Matsumoto, J., Ohshima, T., Isono, O., and Shimotohno, K. (2005). HTLV1 HBZ suppresses AP-1 activity by impairing both the DNA-binding ability and the stability of c-Jun protein. Oncogene 24, 1001-1010.

Matsuoka, M., and Jeang, K. T. (2007). Human T-cell leukaemia virus type 1 (HTLV-1) infectivity and cellular transformation. Nat. Rev. Cancer 7, 270-280.

Nicot, C., Harrod, R. L., Ciminale, V., and Franchini, G. (2005). Human T-cell leukemia/lymphoma virus type 1 nonstructural genes and their functions. Oncogene 24, 6026-6034.

Poiesz, B. J., Ruscetti, F. W., Gazdar, A. F., Bunn, P. A., Minna, J. D., and Gallo, R. C. (1980). Detection and isolation of type $\mathrm{C}$ retrovirus particles from fresh and cultured lymphocytes of a patient with cutaneous T-cell lymphoma. Proc. Natl. Acad. Sci. U.S.A. 77, 7415-7419.

Saito, M., Matsuzaki, T., Satou, Y., Yasunaga, J., Saito, K., Arimura, K., Matsuoka, M., and Ohara, Y. (2009). In vivo expression of the HBZ gene of HTLV-1 correlates with proviral load, inflammatory markers and disease severity in HTLV-1 associated myelopathy/tropical spastic paraparesis (HAM/TSP). Retrovirology 6, 19.

Satou, Y., Yasunaga, J., Yoshida, M., and Matsuoka, M. (2006). HTLV-I basic leucine zipper factor gene mRNA supports proliferation of adult $\mathrm{T}$ cell leukemia cells. Proc. Natl. Acad. Sci. U.S.A. 103, 720-725.

Satou, Y., Yasunaga, J., Zhao, T., Yoshida, M., Miyazato, P., Takai, K., Shimizu, K., Ohshima, K. Green, P. L., Ohkura, N., Yamaguchi, T., Ono, M., Sakaguchi, S., and Matsuoka, M. (2011). HTLV1 bZIP factor induces T-cell lymphoma and systemic inflammation in vivo. PLoS Pathog. 7, e1001274. doi: 10.1371/journal.ppat.1001274

Suemori, K., Fujiwara, H., Ochi, T., Ogawa, T., Matsuoka, M., Matsumoto, T., Mesnard, J. M., and Yasukawa, M. (2009). HBZ is an immunogenic protein, but not a target antigen for human T-cell leukemia virus type 1-specific cytotoxic T lymphocytes. J. Gen. Virol. 90, 1806-1811.

Sugata, K., Satou, Y., Yasunaga, J., Hara, H., Ohshima, K., Utsunomiya, A. Mitsuyama, M., and Matsuoka, M. (2012). HTLV-1 bZIP factor impairs cell-mediated immunity by suppressing production of Th1 cytokines. Blood 119, 434-444.

Thebault, S., Basbous, J., Hivin, P., Devaux, C., and Mesnard, J. M. (2004). HBZ interacts with JunD and stimulates its transcriptional activity. FEBS Lett. 562, 165-170.

Uchiyama, T., Yodoi, J., Sagawa, K., Takatsuki, K., and Uchino, H. (1977). Adult T-cell leukemia: clinical and hematologic features of 16 cases. Blood 50, 481-492.

Usui, T., Yanagihara, K., Tsukasaki, K., Murata, K., Hasegawa, H., Yamada, Y., and Kamihira, S. (2008). Characteristic expression of HTLV-1 basic zipper factor (HBZ) transcripts in HTLV-1 provirus-positive cells. Retrovirology 5,34 .

Vanhee-Brossollet, C., Thoreau, H., Serpente, N., D'Auriol, L., Levy, J. P., and Vaquero, C. (1995). A natural antisense RNA derived from the HIV-1 env gene encodes a protein which is recognized by circulating antibodies of $\mathrm{HIV}^{+}$individuals. Virology 206, 196-202.
Yamaoka, S., Inoue, H., Sakurai, M., Sugiyama, T., Hazama, M., Yamada, T., and Hatanaka, M. (1996). Constitutive activation of NF-kappa B is essential for transformation of rat fibroblasts by the human T-cell leukemia virus type I Tax protein. EMBO J. 15, 873-887.

Yan, P., Fu, J., Qu, Z., Li, S., Tanaka, T., Grusby, M. J., and Xiao, G. (2009). PDLIM2 suppresses human T-cell leukemia virus type I Tax-mediated tumorigenesis by targeting Tax into the nuclear matrix for proteasomal degradation. Blood 113, 4370-4380.

Yoshida, M., Satou, Y., Yasunaga, J., Fujisawa, J., and Matsuoka, M. (2008). Transcriptional control of spliced and unspliced human T-cell leukemia virus type 1 bZIP factor (HBZ) gene. J. Virol. 82, 9359-9368.

Zhao, T., Satou, Y., Sugata, K., Miyazato, P., Green, P. L., Imamura, T., and Matsuoka, M. (2011). HTLV-1 bZIP factor enhances TGF-beta signaling through p300 coactivator. Blood 118, 1865-1876.

Zhao, T., Yasunaga, J., Satou, Y., Nakao, M., Takahashi, M., Fujii, M., and Matsuoka, M. (2009). Human Tcell leukemia virus type 1 bZIP factor selectively suppresses the classical pathway of NF-kappaB. Blood 113, 2755-2764.

Conflict of Interest Statement: The authors declare that the research was conducted in the absence of any commercial or financial relationships that could be construed as a potential conflict of interest.

Received: 10 May 2012; accepted: 19 June 2012; published online: 09 July 2012.

Citation: Zhao $T$ and Matsuoka $M$ (2012) HBZ and its roles in HTLV-1 oncogenesis. Front. Microbio. 3:247. doi: 10.3389/fmicb.2012.00247

This article was submitted to Frontiers in Virology, a specialty of Frontiers in Microbiology.

Copyright (c) 2012 Zhao and Matsuoka. This is an open-access article distributed under the terms of the Creative Commons Attribution License, which permits use, distribution and reproduction in other forums, provided the original authors and source are credited and subject to any copyright notices concerning any third-party graphics etc. 